\title{
POSITRON ANNIHILATION IN AND COMPRESSIBILITY OF WATER-ORGANIC SOLUTIONS. THE SYSTEM WATER-PYRIDINE
}

\author{
K. JeRIE, A. Baranowski \\ Institute of Experimental Physics, University of Wrocław \\ Cybulskiego 36, 50-205 Wrocław, Poland \\ J. Gliński and B. Jeżowska-Trzebiatowska \\ Institute of Chemistry, University of Wrochaw \\ F. Joliot-Curie 14, 50-383 Wrocław, Poland
}

(Received April 17, 1992)

\begin{abstract}
The results of positron lifetime and ultrasonic velocity measurements in dilute aqueous pyridine (PYR) solutions show unusual changes in ultrasonic, annililation data in function of pyridine concentration. The results obtained suggest that strong structural changes occur in the system, in the region of low pyridine concentrations. Liquid clathrate hydrates forming in the system are supposed with unusual stoichiometry and untypical geometry. They are supposed to be very short-living and/or unstable.
\end{abstract}

PACS numbers: $61.25 . \mathrm{Em}, 78.70 . \mathrm{Bj}$

\section{Introduction}

In the present work using pyridine as solute, we have tried to compare the ultrasonic and positron annililation behaviour of this system. This paper is a continuation of the series $[2,3,4,18]$, in which we show that different and independent experimental methods give the results which can be interpreted using the same physical picture of the structure of liquid. 


\section{Experimental}

\subsection{Malerials}

Pyridine pure for synthesis (POCh, Gliwice, Poland) was purified by fractional distillation. Water was doubly distilled and chemicals freshly prepared were used for measurements. The concentrations of the solutions were determined by weighing the components.

\subsection{Density measurements}

Density was measured using a calibrated sinker method at the temperature range of $15-35^{\circ} \mathrm{C}$. Calibrations were determined daily using the known densities of air and $\mathrm{H}_{2} \mathrm{O}$. The reproductibilities of the densities were $\pm 0.1 \mathrm{~kg} / \mathrm{m}^{3}$.

\subsection{Sound velocily measurements}

The ultrasonic velocity was measured with an accuracy of $\pm 0.1 \%$ using a sing-around equipment. The details of the apparatus and measurement techniques applied in this study are given in [1].

\subsection{Posilron annihilation measurements}

The annihilation measurements were carried out at room temperature (approx. $25^{\circ} \mathrm{C}$ ) with a standard measuring device based on the "fast-slow" coincidence technique and described elsewhere [2].

\section{Experimental results}

\subsection{Ultrasonic properties of the system waler-pyridine}

The adiabatic compressibility coefficients $\beta$ were calculated from Laplace's equation

$$
\beta=\left(d c^{2}\right)^{-1},
$$

where $c$ is the ultrasonic velocity and $d$ - the density of the liquid. The concontration dependence of the compressibility within the low pyridine concentration region, which is of interest for the further discussion, is shown in Fig. 1.

The adiabatic compressibility plotted against the mole fraction of pyridine shows that in the concentration range covered by our measurements the isotherms intersect approximately at a common point corresponding to the concentration $X_{\text {PYR }}=0.045$. However, in contrary to the systems investigated beforc, the concentrational dependence of compressibility is not smooth and its shape depends strongly on temperature. It reminds the system water-cyclohexylamine [3], in which considerable anomalies of the sound velocity and adiabatic compressibility at low amine concentrations were found. 


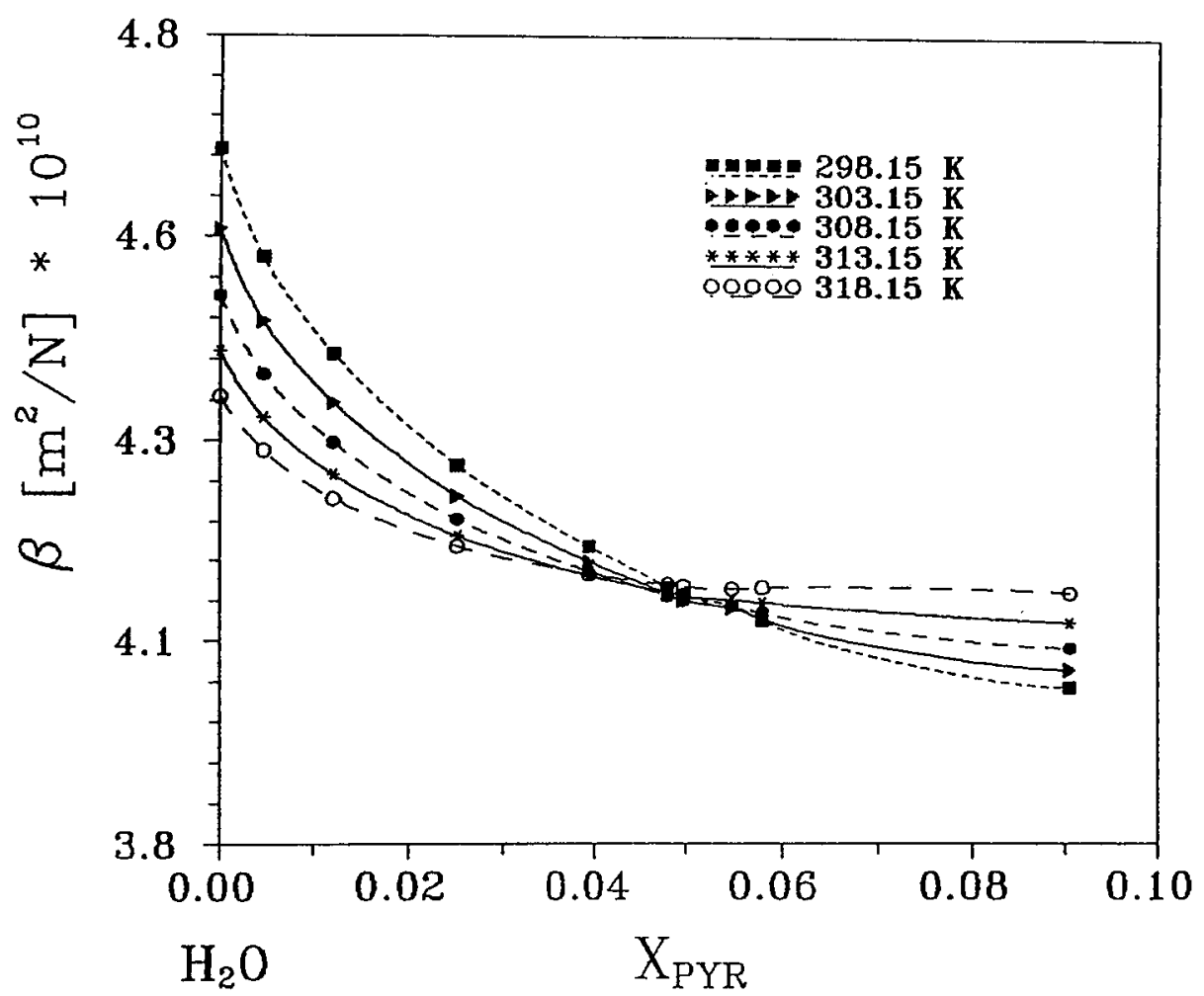

Fig. 1. Dependence of the adiabatic compressibility coefficient on the pyridine concentration.

\subsection{Positron mean lifetimes}

All the details of treatment of the annihilation data are given in details in other papers of this series [2, 3, 18], also in the most recent one [4]. Finally, the positron annihilation spectrum was separated into two components: a short-lived one $\tau_{1}$ with intensity $I_{1}$, connected with the free positron annihilation and with the para-positronium, and a long-lived component $\tau_{2}$ with intensity $I_{2}$, distinctly separated from the former one. The latter one, of intensity $I_{2}$, was attributed to ortho-positronium annihilating entirely in the pick-off process [5-7]. The short lifetime component (with subscript $\tau_{1}$ ) is due to the free positron annihilation and to para-positronium annihilation. In this treatment the following condition is fulfilled

$$
I_{1}+I_{2}=100 \% \text {. }
$$

From the analysis of the time spectrum of the pyridine solutions the decay constants $\lambda_{1}=\tau_{1}^{-1}$ and $\lambda_{2}=\tau_{2}^{-1}$ were determined. The results are presented in Fig. 2. 


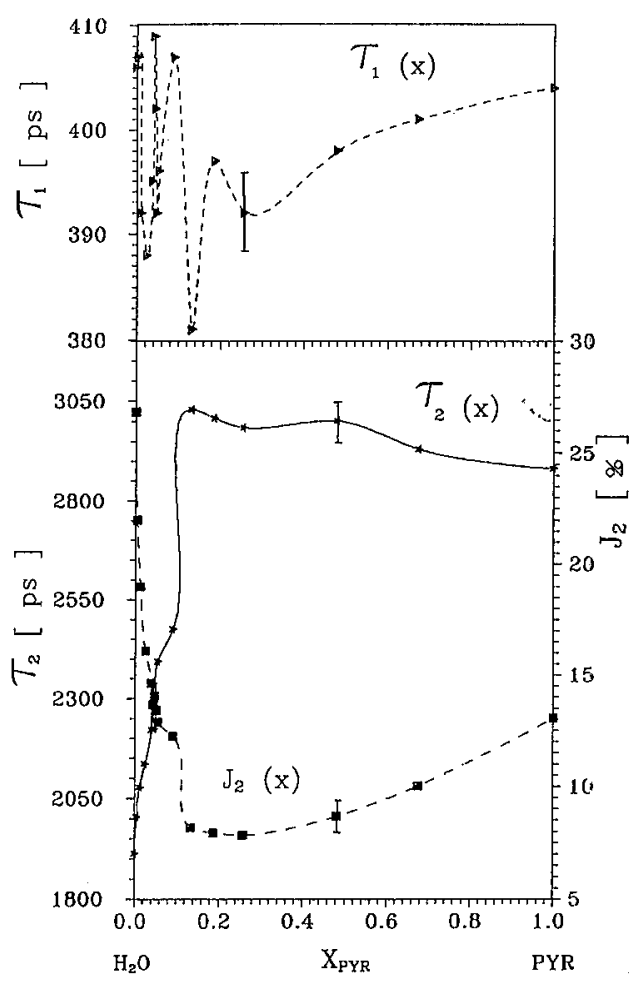

Fig. 2. The results of positron annihilation measurements: $\tau_{1}$ - lifetimes of the short-lived component, $\tau_{2}$ - lifetimes of the long-lived component, $I_{2}$ - intensity of the latter vs. molar fraction of pyridine.

\section{Discussion}

\subsection{Adiabalic compressibility}

To the best of our knowledge, the formation of clathrate hydrates of pyridine has not been reported in literature including the most representative reviews on clathrate type compounds $[8,9]$. This suggests that either pyridine does not form inclusion compounds of this type with water or they are too unstable to be isolated.

According to the concept of iceberg formation of Frank and Evans [10] or to that of hydrophobic hydration formulated by Franks [11], when an organic compound is dissolved in water, the watcr structure is modified around the nonpolar parts of the solute molecule in the direction of greater "crystallinity", whatever the actual quasi-solid water structure is induced in this way. Thus, the water structure in the vicinity of an organic molecule is determined mainly by the hydrophobic moiety of the guest, the latter being accommodated in the cavities of the open hydrogen-bonded water network, which in pure water are occupied most probably by "monomeric" water molecules [12-15]. This physical picture seems to give a 
reasonable interpretation of some properties of non-electrolyte aqueous solutions, e.g. of p-dioxan [16], tetrahydrofuran [17], ethanol [18] and some amines [19].

According to Endo [20], the intersection point of the adiabatic compressibility isotherms should be attributed to the formation of clathrate-like structures in liquid water-organic systems at low concentrations of the organic component. A similar structural interpretation of the concentration dependence of compressibility of aqueous solutions of non-electrolytes was suggested in [19] and [21]. Thus, the intersection point concentration indicates a clathrate stoichiometry of 1 PYR : $21.2 \mathrm{II}_{2} \mathrm{O}$.

In solid state most of the water-soluble polar compounds form cubic lattices of two types: I of $\mathrm{CsCl}$ type (with a lattice dimension $a=1.2 \mathrm{~nm}$ ) and II of diamond type (with a lattice dimension $a=1.7 \mathrm{~nm}$ ) [8-9, 22-23]. The details of these structures are given in the recent papers of this series $[2,3,4,18]$.

The crossing point concentration $X_{P Y R}=0.045$ does not correspond to any of the known structures. However, the geometry of pyridine molecule is not as simple as that of the compounds investigated by us before (simple alcohols and amines). Due to this geometry, it is possible, for example, that one pyridine molecule forms with water a quasi-clathrate structure which is not known in solid state. It is worth noting that the compressibility value at the crossing point $(4.12 \times$ $10^{-10} \mathrm{~m}^{2} \mathrm{~N}^{-1}$ ) is much higher than that found by von Stackelberg for the solid clathrates of structure II $\left(4.0 \times 10^{-10} \mathrm{~m}^{2} \mathrm{~N}^{-1}\right)$ [22, 23].

As the pyridine concentration is increased upwards of $X_{P Y R}=0.045$, the anomalous compressibility dependence on concentration of the organic solute appears. The increasing water shortage should lead to the formation of larger water cavities [23], in which two or even more organic molecules associated in a hydrophobic sense may be accommodated. Those clathrate structures may exist in a temperature dependent equilibrium. The accommodation of two organic molecules in a common water envelope at organic component concentrations higher than 0.2 was suggested by Gorbunov et al. [24] on the basis of IR and NMR spectra of the water-dioxane system. A variety of structures existing simultaneously in equilibrium seems to be supported also by the results of freezing point measurements reported by Ennan and Lapshin [25] for the system water-tetrahydrofuran.

Ilowever, such an interpretation of the adiabatic compressibility data is possible if the compressibility isotherms, after intersecting at a common point, achieve minima in concentrations depending on temperature. In the case of water-pyridine system the situation is quite different, the concentrational dependencies of the compressibility are anomalous and do not demonstrate the distinct minima. Consequently, the interpretation given above being valid for most of the water-organic liquid mixtures is not useful for the water-pyridine system.

There are many possible reasons for the unusual behaviour of this system. It is possible, for example, that the hydrophobic part pf the pyridine molecule is too big to be accomodated in the water cavities, or that the significant hydrophilic hydration takes place, because of the charge distribution all over the molecule of pyridine. So far, we are not able to choose between these interpretations.

Concluding, the structure of dilute solutions of pyridine in water is not typical. It does not correspond to the two structures known for solid clathrates. The 
above interpretation was made mainly by comparison of the ultrasonic properties of the system under investigation with the systems investigated before. The ultrasonic velocity data give not enough information for proper interpretation and one needs more experimental data for it.

\subsection{Positron annihilation}

The concentration dependencies of positron annililation parameters remind a bit that of the water-alcohol systems [26]. The concentrations of the $I_{2}$ and $\tau_{2}$ deflections are close to the concentration of the organic solute corresponding to the intersection point of compressibility isotherms. The results indicate a remarkable structural variation with composition in the water rich region. In this system different structures are probably formed, involving the hydrate structures of clathrate type with significant perturbations due to untypical geometry and/or interactions of the pyridinc molecule. Ilowever, the concentration dependence of $\tau_{1}$ is very untypical and complicated and does not remind any other system investigated before.

Usually only the long-lived parameters, the intensity $I_{2}$ and lifetime $\tau_{2}$, are interpreted in terms of liquid structure. The annihilation parameters of the pick-off process of an ideal mixture of classical liquids are a simple function of concentration [2]

$$
\lambda_{2 \mathrm{AB}}^{1 / 2} V_{\mathrm{AB}}=\lambda_{2 \mathrm{~A}}^{1 / 2} V_{\mathrm{A}}-\left(\lambda_{2 \mathrm{~A}}^{1 / 2} V_{\mathrm{A}}-\lambda_{2 \mathrm{~B}}^{1 / 2} V_{\mathrm{B}}\right) X_{\mathrm{B}}
$$

where $\lambda_{2 A B}$ - the ortho-positronium decay constant in the pick-off process in the mixture of liquids $A$ and $B, \lambda_{2 A}$ and $\lambda_{2 B}$ - the corresponding quantities for the pure liquids $\mathrm{A}$ nad $\mathrm{B}, V_{\mathrm{A}}$ and $V_{\mathrm{B}}-$ molar volumes of the components $\mathrm{A}$ nad $\mathrm{B}$, $X_{\mathrm{B}}$ - molar fraction of the $\mathrm{B}$ component. $V_{\mathrm{AB}}-$ the mean molar volume of the mixture $\mathrm{A}-\mathrm{B}$

$$
V_{\mathrm{AB}}=\left(X_{\mathrm{A}} M_{\mathrm{A}}+X_{\mathrm{B}} M_{\mathrm{B}}\right) / d
$$

where $M_{\mathrm{A}}$ - molecular mass of water, $M_{\mathrm{B}}$ - molecular mass of pyridine, $d-$ the measured density of the mixture.

Taking into consideration the formula (3) and the positron annihilation data, we obtained the values of "ideal" $\lambda_{2 \mathrm{AB}}$. Through the relation (3) $\lambda_{2 \mathrm{AB}}$ is connected with $d(X)$. The smooth dependence of $d(X)$ results in a smooth concentration dependence of $\lambda_{2 \mathrm{AB}}$. The experimental values of $\lambda_{2 \mathrm{AB}}$ differ significantly from those obtained from the relation (3). Therefore the latter formula is evidently incorrect for pyridine-water mixtures. The plot of $\Delta V_{\mathrm{AB}}$ (defined as difference between "ideal" and experimental $V_{\mathrm{AB}}$ 's) vs. pyridine content in water shows (Fig. 3) not-monotonical character and inflections in the water-rich region. In the limits of the experimental error it is difficult, however, to decide about the subtle changes in this dependence.

The lifetime of the ortho-positronium is markedly affected by the surface tension coefficient $\sigma$. According to the "bubble" model of the pick-off annihilation process $[27,28]$

$$
{ }^{*} \lambda_{2}=A \sigma^{\gamma}
$$




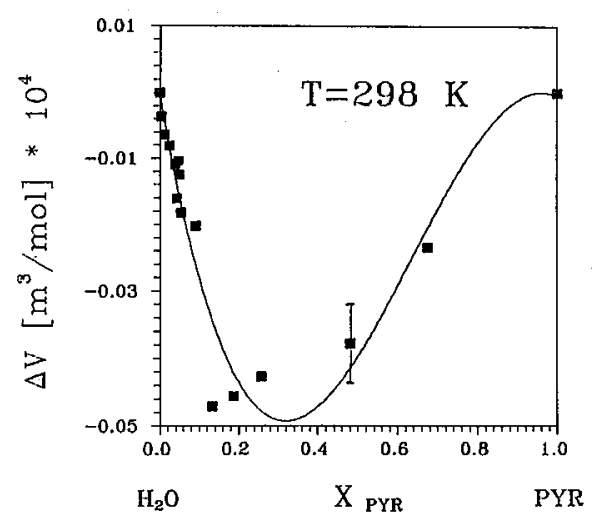

Fig. 3. The "excess" molar volume of pyridine mixtures with water vs. pyridine concentration.

with the $A$ and $\gamma$ parameters fitted so as to obtain ${ }^{*} \lambda_{2}=\lambda_{2}$ for $X=0$ and $X=1$. We measured the surface tension coefficients at $298.15 \mathrm{~K}$ and calculated the $A$ and $\gamma$ parameters. They are $A=0.03469$ and $\gamma=0.63304$. The similarity of the concentration dependence of $\lambda_{2}$ to that of * $\lambda_{2}$ calculated from Eq. (5) confirms, at least qualitatively, this annihilation model. If it is assumed that the surface tension is the main lifetime controlling factor in the pick-off annihilation, the difference $\Delta \lambda_{2}={ }^{*} \lambda_{2}-\lambda_{2}$ may be attributed to structural effects. The dependence of $\Delta \lambda_{2}$ on pyridine concentration is shown in Fig. 4. The extremely complicated character of this dependence, especially in low pyridine concentrations, indicates that strong structural changes occur in the water-pyridine solutions at low $\left(X_{\mathrm{PYR}}<0.2\right)$ concentrations of the organic solute.

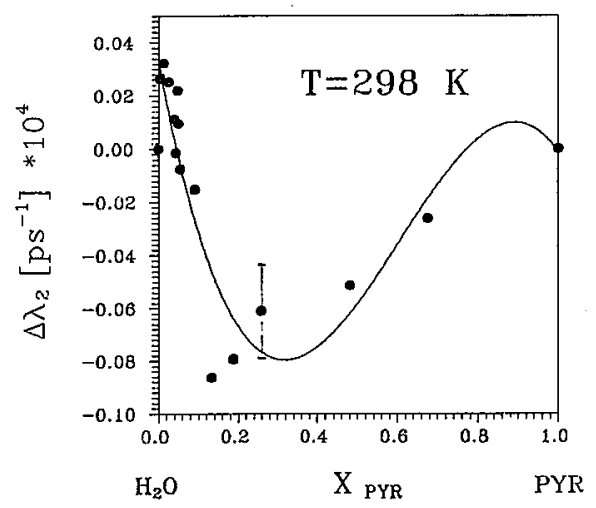

Fig. 4. The "excess" decay constant calculated from surface tension vs. pyridine concentration. 


\section{Conclusions}

The experimental results obtained for the system water-pyridine using two different methods (ultrasonic velocity and positron annihilation) and interpreted independently are both explicable within the framework of liquid pseudo-clathrate structure. The results show that in the system under investigation sharp and strong structural changes occur in the region of low pyridine concentrations. The stoichiometry of the liquid hydrates is approximately 1 PYR : $21.2 \mathrm{II}_{2} \mathrm{O}$ and does not correspond to the most common clathrate structures. It may therefore be concluded that the structure formed by pyridine in its dilute aqueous solutions is different from those known in solid state. Thus, it can be considered as a clathrate hydrate of unusual structure and stoichiometry. Similar unusual structures were reported for some amines (n-prophylamine, diethylamine). This is probably the result of the specific geometry of the pyridine molecule and/or additional hydrophilic hydration of pyridine molecule. The anomalous compressibility dependence on concentration, as well as anomalies in the positron annihilation parameters, allows us to suppose that in the region of low organic component concentrations different quasi-clathrate hydrates are formed. It is possible that hydrophilic hydration also takes place in this system, disturbing markedly the experimental picture of the system. It seems probable (although there is no evidence of it) that while adding pyridine to water, at the first moment classical hydrophobic hydration occurs, which yields intercepting the compressibility isotherms, but later other effects appear (other structures competing with each other, hydrophilic hydration and so on), disturbing the system and complicating it.

\section{References}

[1] S. Ernst, J. Gliński, B. Jezowska-Trzebiatowska, Acta Phys. Pol. A55, 501 (1979).

[2] K. Jerie, A. Baranowski, B. Rozenfeld, S. Ernst, J. Gliński, Acta Phys. Pol. A64, 77 (1983).

[3] K. Jerie, A. Baranowski, B. Rozenfeld, S. Ernst, B. Jeżowska-Trzebiatowska, J. Gliński, Acta Phys. Pol. AG6, 3 (1981).

[4] K. Jerie, A. Baranowski, B. Rozenfeld, B. Jeżowska-Trzebiatowska, J. Gliński, Acta Phys. Pol. A79, 507 (1991).

[5] B. Levay, A. Vertes, P. Irautojarvi, J. Phys. Chem. 77, 2229 (1973).

[6] B. Levay, P. Hautojarvi, Radiochem. Radioanal. Lett. 10, 309 (1972).

[7] R.E. Wild, II.J. Ache, Radiochem. Radioanal. Lett. 23, 249 (1975).

[8] G.A. Jeffrey, R.K. McMullan, Prog. Inorg. Chem. 8, 43 (1967).

[9] S.Sh. Byk, Ju.F. Makogon, V.I. Fomina, Gazovye Gidrati, Izd. Khim., Moscow 1980.

[10] H.S. Frank, M.W. Evans, J. Phys. Chem. 13, 507 (1945).

[11] F. Franks, Ann. N. Y. Acad. Sci. 125, 277 (1965).

[12] L. Pauling, in: Hydrogen Bonding, Eds. D. Hadzi, H.W. Thompson, Pergamon, London 1959 , p. 1.

[13] H.S. Frank, A.S. Quist, J. Chem. Phys. 34, 604 (1961). 
[14] V.N. Yashkistshev, O.Ya. Samoilov, Zh. Strukt. Khim. 3, 211 (1962).

[15] M.N. Buslaeva, O.Ya. Samoilov, Zh. Slrukt. Khim. 4, 502 (1963).

[16] R. Savickas, A. Tamasauskas, K. Paulauskas, J. Ciparis, Ultragarsas 4, 101 (1972).

[17] S. Ernst, J. Gliński, Chem. Tech. 29, 51 (1977).

[18] K. Jerie, A. Baranowski, S. Ernst, J. Gliński, Acta Phys. Pol. A69, 81 (1986).

[19] S. Ernst, J. Gliński, Polish J. Chem. 56, 339 (1982).

[20] H. Endo, Bull. Chem. Soc. Jpn. 16, 1586 (1973).

[21] S. Ernst, J. Gliński, Mater. Sci. 3, 68 (1977).

[22] H. Müller, M. von Stackelberg, Naturwissenschaften 39, 20 (1952).

[23] M. von Stackelberg, H. Müller, Z. Elektrochem. 58, 25 (1954).

[24] B.Z. Gorbunov, V.S. Kozlov, U.I. Nabyerukhin, Zh. Strukt. Khim. 16, 816 (1975).

[25] A.A. Ennan, V.A. Lapshin, Zh. Fiz. Khim. 49, 2295 (1975).

[26] K. Jerie, A. Baranowski, B. Rozenfeld, J. Gliński, S. Ernst, Phys. Scr. 35, 729 (1987).

[27] A.B. Buchikhin, V.J. Goldanskii, A.D. Tatur, V.P. Shantarovitch, Zh. Eksp. Teor. Fiz. 60, 1436 (1971).

[28] S.J. Tao, J. Chem. Phys. 56, 5499 (1972). 